Artículo de investigación

Apuntes del CENES

ISSN 0120-3053

Volumen $35, N^{\circ} 61$

Enero - Junio 2016

Págs. 237-266

\title{
El proceso de sustitución de combustibles pesados por gas natural en el sector industrial del Valle del Cauca y del Cauca - Colombia 2004-2012
}

The process for replacing heavy fuel oil with natural gas in industrial companies of Valle del Cauca and Cauca - Colombia 2004-2012

\author{
Do processo para a substituição de óleo \\ combustivel pesado com a Gas Natural em \\ empresas industriais de Valle del Cauca e \\ Cauca - Colombia 2004-2012
}

Andrés Eduardo Rangel Jiménez* Carlos Johnny Portilla Salazar**

Fecha de recepción: 11 de agosto de 2015

Concepto de evaluación: 26 de noviembre de 2015

Fecha de aprobación: 9 de diciembre de 2015

Magister en Econometría Universidad Torcuato di Tella. Docente de planta, Departamento de Ciencias Económicas de la Universidad Autónoma de Occidente. Cali, Colombia. Código postal: 760030. Correo electrónico: aerangel@uao.edu.co

* Coordinador grandes consumidores. Administrador de Empresas. Especialista en Economia Ambiental. Universidad San Buenaventura Cali, Colombia. Gases de Occidente S.A. ESP. Cali, Colombia. Código postal: 760030. Correo electrónico: carlosjp@gasesdeoccidente.com 


\section{Resumen}

Este documento tiene por objetivo analizar el proceso de sustitución de la utilización de combustibles pesados hacia el gas natural por parte de medianas y grandes empresas ubicadas en el Valle del Cauca y norte del Cauca, que se observan durante el periodo 2004-2012. Asimismo, utilizando un modelo Probit de efectos aleatorios en Panel, se estima la propensión de estas industrias a sustituir un combustible altamente contaminante por gas natural. Los resultados muestran que la diferencia de gasto en gas con relación a otros combustibles es determinante en la probabilidad de conversión. Adicionalmente, se confirma que las industrias con menos probabilidad de realizar la conversión son aquellas que utilizan carbón en los procesos de producción.

Palabras clave: fuentes de energía alternativa, gasificación, datos de panel.

Clasificación JEL: C33, C51, Q420.

\section{Abstract}

This paper has the main purpose to analyze the replacement process from heavy fuel oil toward natural gas by median and big companies located in Valle del Cauca and north of Cauca in the period 2004-2012. Likewise, using a linear probability Panel Data model, the replacement propensity from higher polluting fuel toward natural gas of these industries is estimated. The results show that the difference in spending on natural gas to other fuel usage is determinant to the probability of replacement. In addition, it is confirmed that industries with less likelihood of replacement are those that use coal in production processes.

Keywords: Alternative energy sources, gasification, panel data model. 


\section{Resumo}

Este trabalho tem por objetivo analisar o processo de substituição do uso de óleo combustível pesado para gás natural por empresas de médio e grande porte localizadas no Valle del Cauca e Cauca, Norte del vistos ao longo do período 2004-2012. Além disso, usando um modelo de efeitos aleatórios Painel de Probit, a propensão destas indústrias é estimada para substituir combustíveis altamente poluentes a gás natural. Os resultados mostram que a diferença de passar sobre o gás em relação a outros combustíveis é crucial para a probabilidade de conversão. Em adição,confirmou-se que as indústrias menos susceptíveis de converter são aqueles que usam processos de produção de carvão.

Palavras-chave: Fontes Alternativas de Energia, gaseificação, dados em painel. 


\section{INTRODUCCIÓN}

La energía desempeña un papel crucial en el sector industrial y en el de consumo, cuyo uso produce una serie de externalidades asociadas casi inevitablemente con efectos nocivos para el medio ambiente. Es claro, por otra parte, que la mayoría de combustibles utilizados en la industria es de carácter no renovable, lo que hace necesaria su valoración para garantizar su uso a las generaciones futuras. Es esto precisamente lo que lleva a la búsqueda de un uso eficiente de energía, que requiere de una valoración del desempeño de las distintas fuentes de energía dentro del proceso productivo, para así lograr una mejora en los equipos consumidores de recursos energéticos, mediante procesos de sustitución de combustibles.

En este orden de ideas, el concepto de uso racional de la energía (URE) en Colombia toma fuerza en los últimos años, al constituirse en una alternativa orientada a utilizar de forma eficiente la energía eliminando el desperdicio y uso innecesario de la misma. El uso racional y eficiente de las fuentes de energía no solo mejora la eficiencia y la competitividad de las empresas, sino también la calidad del medio ambiente. Precisamente respecto a este último punto el análisis de sustitución pretende, de paso, reducir el impacto ambiental al reducir la emisión de gases de invernadero provenientes de los procesos de combustión altamente contaminantes.

La reducción de emisiones se ha convertido en uno de los desafíos políticos y económicos de los países desarrollados y en vía de desarrollo, como se pone de manifiesto en la lucha contra el cambio climático por muchos tratados y convenios, los cuales inician con la firma del Protocolo de Kioto (diciembre 11 de 1997). 
Si bien durante la década de los noventa las emisiones comunitarias se redujeron en un 3,5\%, los cálculos del año 2010 indicaron que este tipo de contaminación solo disminuyó en un 4,7 \%, es decir, 3,3 puntos por debajo del objetivo del $8 \%$ establecido en el Protocolo de Kioto para el año 2020.

En la actualidad los gobiernos buscan incentivar el uso de combustibles que tengan un relativo menor impacto en las emisiones que descargan a la atmósfera. Un reciente estudio de la Organización Mundial de la Salud (OMS) estima que solamente el $12 \%$ de la población mundial que reside en ciudades respira aire limpio, y casi la mitad convive con una polución 2,5 veces mayor que los niveles recomendados por el organismo. En este orden de ideas, la utilización de gas natural representa un avance en materia ambiental, ya que en sectores como el de transporte e industrial, sustituye principalmente combustibles fósiles con un alto nivel de emisiones.

En este escenario se postula el gas natural como un sustituto viable de combustibles pesados o carbón, o ambos, que permita al sector industrial ser competitivo y producir los artículos que se demanden de manera responsable con el medio ambiente. El gas natural se ha convertido en los últimos años en Colombia en una fuente de energía sustituta de la energía eléctrica y otros combustibles fósiles, llegando a constituirse en un serio respaldo en periodos de escasez, tal como ocurrió durante el fenómeno del Pacífico (El Niño) en 2009 y parte del $2010^{1}$. Como evidencia se recuerda el caso del gasoducto Ballena-Barrancabermeja, cuyo volumen transportado fue llevado a la máxima capacidad de carga, situación explicada principalmente por la alta demanda de las termoeléctricas a gas, originada por los efectos del fenómeno de El Niño.

El objetivo de este trabajo es presentar distintos escenarios en el mercado energético adaptados que muestren eficiencia energética de la industria intensiva en la utilización de diferentes tipos de combustible, con respecto a las emisiones de $\mathrm{CO}_{2}$, rendimiento calorífico y económico. El uso de la herramienta se ilustra mediante la presentación de escenarios de distintas empresas que utilizan diversas fuentes energéticas. Cabe señalar que la herramienta presentada no aborda los pasos que se tienen que seguir para realizar la conversión en las calderas de vapor que queman combustible líquidos a gas. Si bien no pretende ser un modelo completo de análisis del mercado de la energía con cálculos de equilibrio etc.,

\footnotetext{
De acuerdo con el informe Statistical Review of World Energy (British Petroleum BP, 2013), en el año 2012 el gas natural ocupaba el tercer lugar entre las fuentes de energía primaria con un $24 \%$ del total a nivel mundial. Uno de los usos principales se da en las actividades de producción industrial, generación eléctrica o cogeneración
} 
sí intenta ser una primera aproximación, al formalizar el análisis de sustitución de combustibles frente a un vacío existente respecto al tema.

\section{DINÁMICA DEL SECTOR IN- DUSTRIAL EN EL VALLE DEL CAUCA}

La economía del Valle del Cauca difiere de la estructura productiva del país, lo cual puede notarse en el peso de la producción en algunas manufacturas y servicios superior al del promedio del país. El alto nivel de concentración en cada uno de los sectores (en el agro se concentra en caña de azúcar y café, y en la industria en refinerías de azúcar, petróleo y productos químicos), hizo que la economía departamental fuera más vulnerable a la apertura económica específicamente a los choques externos. De hecho, la apertura económica contribuyó a la desaparición de los cultivos agrícolas transitorios y a la tercerización del PIB del departamento.

Es decir, durante ese período, muchas empresas del Valle tuvieron que suspender su producción o cambiar su modo de operación, ya que no podían seguir compitiendo con los precios internacionales. Parte de la reconversión industrial tuvo que ver con este proceso de mejorar la competitividad. En este proceso el sector terciario se favoreció con esta situación, debido a que algunas industrias regionales se volcaron a la comercialización de bienes y empezaron a requerir una nueva estructura económica más enfocada en la distribución de mercancías y operaciones de logística comercial, para satisfacer necesidades que antes no existían en la ciudad y el departamento (Otero, 2012).

En efecto, ya para el año 2010, el PIB del Valle estaba conformado principalmente por actividades del sector terciario $(70 \%)$, específicamente por empresas de servicios, comercio e intermediación financiera. El restante $30 \%$ lo produjeron las actividades del sector secundario (24\%) y el sector primario (6\%). Esta composición ha sido dinámica en el tiempo, pues en 1990 el sector terciario aportaba el $52 \%$ del PIB del Valle, mientras que el secundario (industria y construcción) aportaba el $31 \%$. Diez años después la fuerza de la tercerización se hizo sentir, pues estas cifras pasaron a $73 \%$ y $20 \%$, respectivamente.

Para el último año del estudio, esto es 2012, el PIB departamental por sectores muestra que el sector de servicios financieros aportó el $26.1 \%$, los servicios comunales y sociales el $14.6 \%$ y la industria el $15.5 \%$. Por su parte, el sector agropecuario aporto solo el $5 \%$, y el sector de electricidad, gas y agua el $3.7 \%$ (Otero, 2012). Si se analiza por tasas de cambio se observa que durante el 2007 y el 2012 la estructura económica estuvo marcada por una clara especialización en el sector comercio. En efecto, si se analizan por sectores las tasas de crecimiento, se 
notará que el de mayor dinamismo fue el sector comercio (4,4\%), seguido del minero $(4,3 \%)$ y establecimientos financieros y actividades de servicios sociales $(3,8 \%)$.

Por otra parte, el sector primario está compuesto principalmente por actividades agrícolas (50\% del sector), entre las cuales se destacan los cultivos de caña de azúcar $(95.17 \%$ de los cultivos permanentes), caña panelera $(0.19 \%)$, café $(0.35 \%)$, hortalizas, cereales (maíz, soya y sorgo) y cultivos frutales. En gran proporción los factores que han incidido en el estancamiento de este sector están asociados al incremento de los costos de producción, especialmente los referidos a los precios de algunos insumos agrarios controlados por grandes monopolios, tasas de intereses onerosas con el sector bancario y altos costos de la energía como el diésel y la energía eléctrica, los cuales están por encima del promedio nacional.

Debe anotarse que el departamento del Cauca, a pesar de constituirse en uno de los principales escenarios del conflicto armado, hoy es un importante polo de desarrollo económico, especialmente en su zona norte, donde se asientan unas 2300 empresas entre grandes, pequeñas y micro. Esta transformación se logró gracias al estímulo vía exenciones de impuestos declarada en la subregión norte del departamento del Cauca. En la última década la economía del Cauca presentó un desempeño sobresaliente en el contexto nacional, registrando una tasa de crecimiento promedio de $6,5 \%$ entre 2004 y 2013, superior al promedio del país $(4,8 \%)$, contrario al Valle del Cauca el cual registró un crecimiento inferior al promedio nacional. Por último, si bien el departamento del Cauca es uno de los más industrializados del país, la participación del sector industrial dentro del PIB del departamento aún dista de ser la de mayor peso.

\section{VENTAJA COMPETITIVA: MÓ- VIL DE LA RECONVERSIÓN INDUSTRIAL}

Si bien es conocido que la ciencia económica tiene teorías bien desarrolladas sobre la asignación de recursos, surge el interrogante de si existen directrices comparativamente claras que se apliquen a un proceso de reconversión industrial, en este caso referente a la sustitución de fuentes de energía.

En la medida en que la reconversión industrial y la reestructuración implicarán un movimiento hacia una economía que es más eficiente y competitiva, ¿significa que debe caracterizarse por lo que hemos entendido como actividades dotadas con ventaja comparativa? La reestructuración industrial como proceso al perseguir la asignación eficiente de recursos probablemente se basará en la maximización del beneficio o minimización del costo, o ambas, a través de la optimización de las técnicas de producción. 
El objetivo de la reconversión industrial es hacer que la estructura de la industria sea más eficiente y más competitiva a nivel internacional. En este punto lo más recomendable sería poner el énfasis en las actividades en las que el país disfruta de una ventaja comparativa. No obstante, los estudios empíricos revelan que la mayoría del comercio entre los países con recursos similares, es intra en lugar de interindustrial, y los efectos de distribución de los ingresos del comercio han sido mucho menores de lo que se esperaba.

Esto ha llevado a los teóricos del comercio internacional a modificar sus modelos, incorporando elementos de la organización industrial como las economías de escala y otros elementos de la competencia imperfecta, llegando incluso a considerar una práctica tan castigada como el dumping, como un elemento teórico importante dentro de la explicación del comercio internacional. Todos estos elementos forman parte de la teoría de ventaja competitiva de Porter (2010), la cual reconoce que si bien las abundantes dotaciones de recursos físicos o mano de obra barata siguen siendo importantes en la explicación de la ventaja competitiva de determinadas actividades económicas básicas, también enfatiza que estos factores no son suficientes para obtener una ventaja dentro del comercio internacional. Sostiene, además, que el cambio tecnológico constituye un medio de evitar o superar la escasez de los factores reduciendo la importancia de la dotación de recursos de un país en particular. En efecto, los aumentos de productividad son esenciales para la competitividad, lo cual requiere de una reestructuración industrial, como lo es en este caso el desplazamiento hacia fuentes de energía más competitivas como el gas natural. Sin duda, las mejoras tecnológicas son fundamentales para la sostenibilidad de las empresas a medio y largo plazo.

Lo cierto es que muchas empresas no aprovechan la asistencia técnica disponible en el mercado, y responden lentamente y con escepticismo a los beneficios de las nuevas tecnologías, como lo es en este caso la transición a gas natural.

El nuevo paradigma de la ventaja competitiva explica por qué las empresas de algunos países optan por mejores estrategias que los de otros. La importancia de la productividad con la que se emplean algunos factores de producción y la importancia de las innovaciones en los métodos y la tecnología utilizada.

\section{BENEFICIOS DEL USO DEL GAS NATURAL}

El gas natural resulta el combustible más económico en términos de la energía entregada (pesos/MMBtu) a la caldera, lo que implica que el costo de generación de vapor, donde el 
combustible incide más del $80 \%$, se reduce utilizando gas (Chalco, 2005).

El gas natural produce una mejor combustión, gracias a que al estar en estado molecular, sus componentes reaccionan de forma más completa con las moléculas de oxígeno. Se produce una combustión más eficiente, porque requiere menos exceso de aire en el quemador, lo que, a su vez, produce menos $\mathrm{CO}_{2}$ y cero partículas. Lo anterior sucede debido a que los quemadores de gas natural no requieren de vapor, como sí lo requieren los combustibles líquidos, y esto no solo evita el costo de demanda de vapor o electricidad para comprimir el aire, sino que resulta ventajoso para el cumplimiento de normas sobre los límites máximos permisibles de emisiones de gas invernadero y partículas en suspensión que pueden endurecerse en un futuro no muy lejano.

Otra ventaja la constituye el hecho de que al no tener presencia de compuestos de azufre altamente corrosivos (esto es una combustión limpia), resulta menos agresivo con las calderas, lo que se traduce en menores paradas y costos de mantenimiento por ductos corroídos o por limpieza.

El aprovechamiento de la energía se hace más eficientemente, pues al producir menos depósitos de hollín en los tubos y menores inquemados en la combustión, las pérdidas de energía por chimenea son menores y se logra una mayor eficiencia térmica en la caldera (Chalco, 2005). Finalmente, al no requerir almacenamiento, toda vez que el gas se entrega de manera confiable por sistemas de tuberías, su manejo se hace de manera relativamente más segura y confiable al evitarse derrames en tanque, líneas y calentador.

\section{Ventajas del uso del gas en los dife- rentes sectores industriales}

En general, el gas natural es el combustible óptimo para utilizar en industrias que emplean hornos y calderas en los procesos productivos, pues por sus características caloríficas, reemplaza satisfactoriamente a los demás combustibles. Por ejemplo, en la industria del vidrio, en la cual se requieren llamas que permitan la transmisión de energía calórica a la masa de cristal, el gas natural cumple con este requisito además de producir un producto mucho más limpio en relación con otros combustibles.

Respecto al sector de alimentos, el gas natural permite llevar eficientemente procesos de cocimiento y secado, posibilitando además el cumplimiento de normas de buenas prácticas para la manufactura (BMP) e incluso normas ISO para la exportación.

En la industria textil, el uso del gas natural permite un ahorro energético en un intervalo del 20 al $30 \%$, al utilizar procesos de transferencia de calor por convección en contraposición a los 
tradicionales que utilizan fluidos intermedios. Igual ocurre en la industria del vidrio, los productos de este tipo de industria requieren algún grado de limpieza, lo cual se logra con el uso del gas natural. La industria cementera, por su parte, utiliza en sus procesos hornos que alargan su vida útil con el gas, puesto que no se requiere de un mantenimiento continuo. Adicionalmente, basta con ver los alrededores de las plantas de producción para darse cuenta que las cementeras que utilizan carbón en sus calderas ocasionan una gran polución, $\mathrm{y}$, en este sentido, la utilización del gas natural en este sector proporciona grandes beneficios para el medio ambiente (Kozulk, 2004).

En los procesos de fundición de metales, el gas natural tiene características que le permiten ser utilizado en diferentes procesos de calentamiento de metales, no solo en la fusión, sino también en el recalentamiento, sin la necesidad de una preparación previa para su utilización, como calentarlo o pulverizarlo en el caso del carbón, lo cual lo hace más eficiente, limpio y económico en aplicaciones como calderas de vapor, secadores, hornos y calentadores

En todas las industrias mencionadas la utilización de gas natural también ofrece una serie de ventajas. La primera de ellas, la cogeneración, que no es más que la producción conjunta de energía eléctrica y calorífica aprovechable. Esta cogeneración permite cubrir las necesidades energéticas no solo de la planta de producción, sino de todas las instalaciones. Otra ventaja es que al no requerir de tanques de almacenamiento, disminuye los riesgos inherentes al almacenaje. Además, una vez cesa la demanda de calor en los procesos, la combustión de gas puede finalizar instantáneamente, lo que favorece los procesos que requieren cargas variables o intermitentes. Todas estas ventajas permitirán a las empresas que hagan la conversión a gas natural reducir sus costos operativos y de esta manera ser más competitivos (Longwell, 2002).

Para terminar, un punto que no debe olvidarse es que a pesar de que el gas natural constituye una fuente de energía limpia y segura, se deben tener en cuenta ciertas normas de seguridad en lo referente tanto a la manipulación como al mantenimiento de los equipos. Asimismo, se requiere del uso de sistemas automáticos de detección de fugas

\section{Análisis de emisiones}

La combustión de cualquier combustible fósil libera calor que se aprovecha en gran parte en la generación de vapor en las calderas. Los principales productos de la combustión son el $\mathrm{CO}_{2}$ (dióxido de carbono) $\mathrm{H}_{2} \mathrm{O}$ (agua), $\mathrm{CO}$ (monóxido de carbono), $\mathrm{NO}_{\mathrm{x}}$ (óxido de nitrógeno) $\mathrm{SO}_{2}$ (dióxido de azufre). De los anteriores gases, el $\mathrm{CO}_{2}$ es considerado gas de efecto invernadero, mientras que los demás gases son 
considerados como contaminantes atmosféricos. Debe anotarse que el factor de emisión generalmente se expresa como el peso de contaminante emitido por la unidad de peso, volumen, energía o actividad, dependiendo del nivel escogido. Por lo general, las emisiones de gases y partículas derivados de los procesos de combustión de fuentes fósiles, así como la dificultad de combustión, aumentan al pasar de utilizar gases al diésel y de este a los petróleos residuales.

El método de referencia a nivel mundial para estimar las emisiones de $\mathrm{CO}_{2}$ asociadas a las actividades energéticas es el propuesto por el IPCC (Intergovernmental Panel on Climate Change), que consiste en contabilizar el volumen de carbón contenido en los combustibles fósiles que utiliza un país (Lee, 2004). Asumiendo que las emisiones de $\mathrm{CO}_{2}$ dependen básicamente de las características de los combustibles y no de las tecnologías de su aprovechamiento, como es el caso con los otros contaminantes, a continuación se presenta un cuadro comparativo de los distintos combustibles y los factores de emisión ${ }^{2}$.

Tabla 1. Factores de emisión de carbono y por tipo de combustible ${ }^{3}$

\begin{tabular}{|c|c|c|}
\hline Combustible & Estado & Factor de Emisión (Kg C/GJ) \\
\hline Carbón & Sólido & 26.8 \\
\hline Crudo de Petróleo & \multirow{5}{*}{ Líquidos } & 20.0 \\
\hline Diésel & & 22.2 \\
\hline Fuel Oil & & 21.1 \\
\hline Gasolina & & 18.9 \\
\hline Kerosene & & 19.5 \\
\hline Gas propano - GLP & \multirow{2}{*}{ Gas } & 17.2 \\
\hline Gas Natural & & 15.3 \\
\hline
\end{tabular}

Fuente: IPCC (1996).

\footnotetext{
2 Las emisiones son todos los fluidos gaseosos, puros o con sustancias en suspensión; así como toda forma de energía radioactiva, electromagnética o sonora, que emanen como residuos o productos de la actividad humana o natural (por ejemplo: las plantas emitan CO2).

3 Factor de emisión calculado a partir de la ecuación estequiométrica.
} 
El proceso de sustitución de combustibles pesados por gas natural en el sector industrial

Andrés Eduardo Rangel Jiménez • Carlos Johnny Portilla Salazar

Tabla 2. Porcentaje de emisiones por combustible ${ }^{4}$

\begin{tabular}{|c|c|c|}
\hline Unidad de Energía Mbtu & Combustible & Produce C02 \\
\hline \multirow{4}{*}{$14.89 \mathrm{Mbtu}$} & Gas Natural & 1.00 \\
\cline { 2 - 3 } & GLP & 1.02 \\
\cline { 2 - 3 } & Crudo & 1.08 \\
\cline { 2 - 3 } & ACPM & 1.16 \\
\cline { 2 - 3 } & Fuel Oil & 1.21 \\
\cline { 2 - 3 } & Gasolina & 1.23 \\
\cline { 2 - 3 } & Carbón Mineral & 1.72 \\
\cline { 2 - 3 } & Energía Eléctrica & 2.08 \\
\cline { 2 - 3 } & Bagazo & 3.70 \\
\hline
\end{tabular}

Fuente: Gases de Occidente S.A. E.S.P.

Puede observarse que no solamente el gas natural es el combustible con menor factor de emisión no solo de $\mathrm{CO}_{2}$ sino también en la emisión de emisiones de chimenea como $\mathrm{CO}$, $\mathrm{NO}_{\mathrm{x}}, \mathrm{SO} 2$

El contenido de carbón de un combustible líquido o solido (fósil) varía en función de sus propiedades físicas y químicas. En cuanto al gas natural, su factor de emisión depende de la composición del gas, que además del metano puede incluir pequeñas cantidades de etano, propano, butano e hidrocarburos pesados. En este orden de ideas, la emisión depende de la proporción o participación de cada uno. A continuación se evalúa el porcentaje de $\mathrm{CO}_{2}$ en emisiones por tipo de combustible.

El cálculo de las emisiones de $\mathrm{CO}_{2}$ provenientes de la ignición de combustibles fósiles está directamente relacionado con dos parámetros: la cantidad de combustibles consumidos y el contenido de carbono de cada uno de ellos, siendo el bagazo, la energía eléctrica y el carbón los de mayores emisiones.

Las estimaciones dadas en la Tabla 2 se fundamentan en las bases teóricas suministradas por la estequiometría de cada uno de los combustibles, establecida a partir de su composición elemental ${ }^{5}$. Las ecuaciones químicas

4 La BTU es una unidad de energía inglesa (millones de unidades térmicas británicas). Es la abreviatura de British Thermal Unit. Una BTU representa la cantidad de energía que se requiere para elevar en un grado Fahrenheit, la temperatura de una libra de agua en condiciones atmosféricas normales. Un pie cúbico de gas natural emite en promedio 1,000 BTU, aunque el intervalo de valores se sitúa entre 500 y 1,500 BTU.

5 Estequiometría es la ciencia que mide las proporciones cuantitativas o relaciones de masa de los elementos químicos que están implicados en una reacción química. 
que rigen las reacciones de combustión son:

$$
\mathrm{CnHM}+(\mathrm{n}+(\mathrm{m} / 4)) \mathrm{O}_{2} \rightarrow(m / 2) \mathrm{H}_{2} \mathrm{O}
$$

Conociendo la composición elemental del combustible y las correspondientes ecuaciones estequiométricas, es posible calcular la cantidad de los productos obtenidos sobre una base dada de combustible. Tratándose de un análisis puramente teórico, se asume una combustión en condiciones de estequiometría completa, en cuyo caso los productos son únicamente $\mathrm{CO}_{2} \mathrm{y} \mathrm{H}_{2} \mathrm{O}$.

Tomando los consolidados por municipios del Valle del Cauca en el año 2013 del sector industrial, se puede observar la participación de las emisiones que se han dejado de generar gracias a la utilización del gas natural. Si bien a primera vista pareciera que las diferencias no son significativas, realmente las diferencias de emisiones, cuando se consideran grandes volúmenes de combustibles contaminantes, saltan a la vista (véase Tabla 3 ).

Como puede observarse, las diferencias en emisiones al año 2013 a favor de la utilización del gas natural en el
Valle del Cauca son notorias, con un ahorro en emisiones para el total del departamento del orden del $35.1 \%$. Dentro del total de emisiones para el departamento, sobresale la alta participación de la energía eléctrica y el carbón (este último más contaminante).

\section{Análisis calorífico de los diferentes combustibles frente al gas natural}

Una de las variables más importantes al momento de tomar la decisión de sustituir un combustible es su poder calorífico, elemento principal para la combustión. Precisamente a continuación se exponen las características caloríficas de los combustibles sólidos, líquidos y gases, relacionados con la unidad de medida utilizada en el mercado colombiano. Vale anotar que el poder calorífico es la energía que se desprende en la combustión completa de una unidad de masa, de materia o de volumen de un combustible en una mezcla estequiométrica con aire ${ }^{6}$.

El primer paso para la conversión es calcular la cantidad de metros cúbicos $\left(\mathrm{m}^{3}\right)$ que se deben utilizar en el remplazo del combustible actual con la siguiente ecuación ${ }^{7}$.

$$
\boldsymbol{m}^{3} \boldsymbol{g} \boldsymbol{n}=\frac{P C C A}{\text { U.medida }} \times \frac{1 f t^{3}}{1000 \text { Btu }} \times \frac{1 m^{3}}{35.31467 f t^{3}}=\text { Cantidad en } m^{3} \text { de gas natural }
$$

\footnotetext{
El poder calorífico se denomina inferior cuando el agua formada en la combustión se considera en estado vapor con los demás productos de la combustión. Se denomina superior cuando el agua formada se considera en fase líquida (condensada).

$7 \quad$ PC CA = Poder calorífico combustible actual; U. Medida = Unidad de medida utilizada; $=$ pie cubico.
} 
El proceso de sustitución de combustibles pesados por gas natural en el sector industrial

Andrés Eduardo Rangel Jiménez • Carlos Johnny Portilla Salazar

Tabla 3. Resumen de emisiones por combustible por municipio

\begin{tabular}{|c|c|c|c|c|c|c|c|c|c|}
\hline Municipio & $\begin{array}{c}\text { No } \\
\text { Industria }\end{array}$ & АСРCM & Carbon & EE & Fuel Oil & GLP & $\begin{array}{l}\text { Total } \\
\text { Ciudad }\end{array}$ & $\begin{array}{c}\text { Gas } \\
\text { Natural }\end{array}$ & Dif \\
\hline Cali & 122 & 2289342 & N.A & 2094774 & 469134 & 217544 & 5070794 & 3500114 & 1570680 \\
\hline Andalucia & 1 & N.A & N.A & N.A & 3522 & N.A & 3522 & 2911 & 611 \\
\hline Buenaventura & 4 & 1.805 & N.A & N.A & N.A & 437 & 2242 & 1920 & 322 \\
\hline Buga & 20 & 190331 & N.A & N.A & 3.533 & 954746 & 1148610 & 1096243 & 52367 \\
\hline Bugalagrande & 2 & 290832 & N.A & N.A & N.A & 13180 & 304012 & 253279 & 50733 \\
\hline Caicedonia & 1 & N.A & N.A & N.A & 448 & N.A & 448 & 370 & 78 \\
\hline Candelaria & 18 & 2166 & N.A & N.A & 60644 & 12317 & 75127 & 63984 & 11143 \\
\hline Caloto & 6 & 136707 & N.A & & N.A & 120291 & 256998 & 230417 & 26581 \\
\hline Cartago & 6 & N.A & N.A & 2157477 & 372642 & 8310 & 2538429 & 1343364 & 1195065 \\
\hline El Cerrito & 1 & N.A & N.A & N.A & 15244 & N.A & 15244 & 12598 & 2646 \\
\hline Guachene & 2 & N.A & N.A & N.A & N.A & 14793 & 14793 & 14503 & 290 \\
\hline Ginebra & 1 & N.A & N.A & N.A & N.A & 19139 & 19139 & 18763 & 376 \\
\hline Guacari & 2 & N.A & N.A & N.A & 1269 & 2542 & 3811 & 3540 & 271 \\
\hline Jamundi & 6 & 25708 & N.A & 832237 & 21622 & 3797 & 883364 & 457419 & 425945 \\
\hline La Union & 1 & 9941 & N.A & N.A & N.A & N.A & 9941 & 8216 & 1725 \\
\hline Palmira & 39 & 1582646 & N.A & 1039800 & 85835 & 205696 & 2913977 & 2080477 & 833500 \\
\hline Puerto Tejada & 2 & 31118 & N.A & N.A & N.A & 24 & 31142 & 25741 & 5401 \\
\hline Roldanillo & 2 & N.A & N.A & N.A & 944 & N.A & 944 & 780 & 164 \\
\hline San Pedro & 2 & N.A & N.A & N.A & N.A & 4798 & 4798 & 4704 & 94 \\
\hline Sevilla & 2 & N.A & N.A & N.A & N.A & 4068 & 4068 & 3989 & 79 \\
\hline Tulua & 9 & 24562 & N.A & N.A & 144930 & 20108 & 189600 & 159789 & 29811 \\
\hline Villarica & 4 & 14940 & N.A & N.A & 50760 & 19453 & 85153 & 73370 & 11783 \\
\hline Yumbo & 82 & 930799 & 8832789 & 4999614 & 1654197 & 1225091 & 17642490 & 10876433 & 6766057 \\
\hline Santander & 1 & 22354 & N.A & N.A & N.A & N.A & 22354 & 18475 & 3879 \\
\hline Zarzal & 3 & N.A & 356924 & N.A & N.A & 18891 & 375815 & 225839 & 149976 \\
\hline Total & 338 & 5552651 & 9189712 & 11153992 & 2884723 & 2865025 & 31646103 & 20477238 & 11168865 \\
\hline
\end{tabular}

*N.A: Informacion no disponible

Fuente: Gases de Occidente S.A. E.S.P.

La ecuación calcula la cantidad de de gas natural que sustituye otro combustible. Tomando como ejemplo de conversión de ACPM por gas natural, la ecuación calcula la cantidad de $\mathrm{m}^{3}$ de gas natural necesaria para cubrir los requerimientos caloríficos:

$$
\underset{\text { lgalón de } A C P M}{138000 \mathrm{Btu}} \times \frac{1 \mathrm{ft}^{3}}{1000 B T U} \times \frac{1 \mathrm{~m}^{3}}{35.31467}=3.91 \mathrm{~m}^{3}
$$


En este ejemplo para reemplazar un (1) galón de ACPM, con un poder calorífico de $138.000 \mathrm{Btu} / \mathrm{gl}$, se requiere $3.91 \mathrm{~m}^{3}$ de gas natural con un poder calorífico de $1000 \mathrm{Btu} / \mathrm{ft} 3$. La anterior ecuación permite elaborar la siguiente tabla para agilizar los cálculos de los metros cúbicos necesarios para realizar la sustitución de combustibles.

Tabla 4. Poder Calorífico de los distintos combustibles

\begin{tabular}{|c|c|c|}
\hline Combustible & Unidad de Medida & Poder Calorífico \\
\hline ACPM & Btu/Gl & 1.00 \\
\hline Bagazo & Btu/Ton & 1.02 \\
\hline Carbón Mineral & Btu/Ton & 1.08 \\
\hline Crudo & Btu/Gl & 1.16 \\
\hline Energía Eléctrica & Btu/kWh & 1.21 \\
\hline Fuel Oil & $\mathrm{Btu} / \mathrm{Gl}$ & 1.23 \\
\hline Gas Natural & $\mathrm{Btu} / \mathrm{m}^{3}$ & 1.72 \\
\hline GLP & $\mathrm{Btu} / \mathrm{Gl}$ & 2.08 \\
\hline Gasolina & $\mathrm{Btu} / \mathrm{Gl}$ & 3.70 \\
\hline
\end{tabular}

Fuente: Gases de Occidente S.A. E.S.P.

MODELANDO EL PROCESO DE SUSTITUCIÓN DE LA FIRMA

\section{Datos}

Los datos tienen como fuente al proveedor de gas natural para la región suroccidental, Gases de Occidente S.A. E.S.P., contando con una estructura de datos de panel, se tiene información en el periodo de tiempo 20092012 para 338 firmas para el Valle del Cauca y norte del Cauca. La variable dependiente es una variable binaria que indica si la firma ha hecho la conversión a gas natural denominada Status $(=1$ si se convierte a gas natural, $=0$ si no lo hace). El total de la muestra se puede descomponer en empresas reguladas y no reguladas, siendo las primeras las empresas pequeñas, mientras que las no reguladas las constituyen las grandes empresas.

Las empresas de la muestra pertenece a 25 municipios la mayoría de Valle del Cauca y en menor medida la subregión norte del Cauca. Los municipios de la muestra son: Cali, Andalucía, Buenaventura, Buga, Bugalagrande, Caicedonia, Candelaria, Caloto, Cartago, El Cerrito, Guachene, Ginebra, Guacari, Jamundí, La Unión, Palmira, Puerto Tejada, Roldanillo, San Pedro, 
Sevilla, Tuluá, Villarrica, Yumbo, Santander de Quilichao y Zarzal. La base de datos consiste de empresas tanto reguladas como no reguladas que en algún momento $t$ del periodo de tiempo de estudio, hayan hecho la transición de combustibles acpm, carbón, energía eléctrica, fuel oil y GLP a gas natural. Hay que anotar que si bien en la base de datos se cuenta con empresas del sector industrial, no se cuenta con información de código CIIU y mucho menos si estas han cambiado de sector. La base de datos no cuenta con esta información debido a la política de privacidad de la empresa Gases de Occidente.
La información se encuentra disponible para las 338 empresas a lo largo del periodo 2009-2012, constituyendo un panel balanceado, que consiste de empresas que realizaron la conversión a gas natural como de aquellas que no realizaron tal conversión. La base ha sido tratada previamente por la Empresa Gases de Occidente de manera que no existe missing en la misma. Dado el compromiso sobre la confidencialidad de la información, solo existe información sobre un consecutivo (número asignado a la empresa $\mathrm{X}$ ) y no sobre su nombre comercial.

Tabla 5. Conversiones de los distintos tipos de fuentes de energía a gas natural

\begin{tabular}{|c|c|c|c|}
\hline Combustible & Unidad de Medida & Factor Multiplicador & \multirow{2}{*}{ Obtiene } \\
\hline ACPM & Galón & 3,900772 & \multirow{2}{*}{} \\
\hline Bagazo & Kilogramo & 0,21804 & \\
\hline Carbón Mineral & Libra & 0,622971 & \multirow{2}{*}{$\mathrm{m}^{3}$ de Gas Natural } \\
\hline Crudo & Galón & 4,190892 & \\
\hline Energía Eléctrica & kWh & 0,096617 & \\
\hline Fuel Oil & Galón & 4,247527 & \\
\hline GLP & Galón & 2,605150 & \\
\hline Gasolina & Galón & 3,267764 & \\
\hline
\end{tabular}

Fuente: Gases de Occidente S.A. E.S.P.

Modelando la probabilidad de que una empresa realice su transición a gas natural, se escogieron como variables explicativas el tipo de combustible utilizado antes de la conversión en el periodo . Incluyendo 5 alternativas de combustibles a saber: ACPM, GLP, carbón, fuel oil y energía eléctrica. Se construyen 5 variables dicotómicas para modelar estas cinco alternativas de combustible, tomando el valor de 1 si pertenecen al combustible i y cero para los demás $\mathrm{j}$ combustibles. La inclusión de estas variables permitirá conocer la probabilidad de convertirse a gas natural, dado el combustible utilizado en el momento. 
Considerado como un consenso, las decisiones de los empresarios se basan en los costos de producción, incluye la variable Diftari, definida en miles de pesos, es la diferencia de tarifas de combustibles alternativos frente a la tarifa del gas natural. Finalmente, la variable emisiones (de $\mathrm{CO}_{2}$ medido en toneladas) es incluida para cuantificar el efecto de esta variable sobre la probabilidad de hacer la conversión.

El ahorro, definido como la diferencia de tarifas, podría considerarse conservador, pues no se consideran otros ahorros por calentamiento de residuales, aditivos, mermas, bombeo o mantenimiento del sistema, cuando se utilizan los combustibles alternativos al gas natural. Sin embargo, al no contar con la información sobre estas variables, se incluye la diferencia de tarifas con el objetivo de identificar probabilidades de sustitución según el combustible que utilizaba antes de la transición a gas natural.

\section{Modelos de elección binaria lineales}

Este apartado considera modelos en los cuales la variable de respuesta en la estructura de panel toma valores de 1 y 0 , una variable dependiente binaria. Modelar la decisión de que una empresa se convierta a gas natural es modelar la decisión de un agente racional que maximiza una función de beneficios, sujeto a restricciones que lo llevan a decidir si realiza o no la conversión. Construyendo variables latentes como estrategia de elección y teniendo presente que la elección debe ser función de características del agente, se tiene el siguiente esquema:

$$
\begin{aligned}
& y_{i t}^{*}=x_{i t} \beta+c_{i}+u_{i t} \\
& y_{i t}=1\left[y_{i t}^{*}>0\right] \\
& y_{i t}=0\left[y_{i t}^{*} \leq 0\right]
\end{aligned}
$$

Siendo la propensión a la conversión que no es observable, estos modelos permiten relacionar variables latentes (no observables) en función de características observables de las empresas, el vector de variables explicativas.

La transición hacia los modelos Probit y Logit se justifica por los problemas que enfrentan los modelos de probabilidad lineal, como lo son la heterocedasticidad y las probabilidades no acotadas entre 0 y 1 .

\section{Modelos de elección binaria no li- neales: Probit y Logit}

Se aborda el tratamiento de la elección de la empresa utilizando modelos Logit y Probit que permiten trabajar con especificaciones no lineales. Usando el esquema de variable latente:

$$
\begin{aligned}
& \mathrm{y}^{*}{ }_{\mathrm{it}}=\mathrm{x}_{\mathrm{it}} \beta+\mathrm{c}_{\mathrm{i}}+\mathrm{u}_{\mathrm{it}} \\
& \mathrm{y}_{\mathrm{it}}=1\left[\mathrm{y}^{*}{ }_{\mathrm{it}}>0\right] \\
& \operatorname{Pr}\left(\mathrm{y}_{\mathrm{it}}=1 \mid \mathrm{x}_{\mathrm{it}} \mathrm{c}_{\mathrm{i}}\right)=G\left(\mathrm{x}_{\mathrm{it}} \beta+\mathrm{c}_{\mathrm{i}}\right)
\end{aligned}
$$


En caso de que la existencia del término de heterogeneidad no observable puede estar relacionada con las variables explicativas, ocasiona que los estimadores no tengan las propiedades deseables.

Como solución alternativa aparece del modelo Logit de efectos fijos, que permite eliminar el término de la ecuación una vez se condiciona sobre lo que a veces se refiere como "estadístico suficiente mínimo" para (Wooldridge, 2005).

Mientras el modelo tradicional Logit de efectos aleatorios requiere estricta exogeneidad, el modelo Logit de efectos fijos relaja el supuesto de estricta exogeneidad. Sin embargo, este modelo no puede obtener estimaciones consistentes de y por lo tanto no puede calcular los efectos marginales convencionales. Esto último constituye un gran inconveniente, dada la necesidad de obtener los efectos marginales pues al ser modelos no lineales los estimadores inicialmente obtenidos carecen de alguna interpretación. En este orden de ideas el modelo Panel Logit con efectos fijos es descartado (Hsiao, 1986).

Como alternativa de estimación se utiliza el tradicional modelo Probit con efectos aleatorios. Esta metodología establece una serie de supuestos del término de error con el término de error inobservable entre otros:

- $c_{\mathrm{i}} \mathrm{y} x_{\mathrm{it}}$ son independientes.
- $x_{\text {it }}$ es estrictamente exógena (lo cual es necesario para especificar la función de verosimilitud cuando se observa una serie de salidas y tratarlas como el producto de factores de verosimilitud individuales.

- $c_{\mathrm{i}}$ tiene una distribución normal con media 0 y varianza

- $y_{\text {it }} \ldots y_{\text {iT }}$ son independientes condicionales sobre $\left(x_{\mathrm{it}}, c_{\mathrm{i}}\right)$

Puede observarse que los supuestos son restrictivos, especialmente porque la endogeneidad en las variables explicativas se descarta. La única ventaja del modelo Probit con efectos aleatorios sobre un modelo Probit combinado (pooled) es que permite correlación en los factores no observables $\left(\mathrm{C}_{\mathrm{i}}, \mathrm{U}_{\mathrm{it}}\right)$.

Si bien en los modelos lineales es fácil eliminar el componente $\mathrm{C}_{\mathrm{i}}$ ya sea utilizando primeras diferencias o con la transformación "within", esas opciones no están disponibles para un modelo no lineal como el Probit, en el que no es posible la diferenciación. Esta es la razón por la cual no existe la opción de estimar directamente con un modelo Probit con efectos fijos (Harris \& Rogers, 2003).

Por otra parte, si se procede a estimar $C_{i}$ directamente adicionando $N-1$ variables dicotómicas por cada observación (empresa), ocasionaría un sesgo en los estimadores, a menos que $T$ sea grande, lo cual no ocurre en este caso. Esto es conocido como el problema de parámetros incidentales: con un $T$ pequeño, la 
estimación de $C_{i}$ es inconsistente, pues un incremento de no $N$ remueve el sesgo (igual sucede en el modelo Logit). Es por esta razón que la estimación del término modelo Probit vía variables dicotómicas es descartado en favor del modelo Probit de efectos aleatorios, como se estableció inicialmente

La especificación del modelo Probit con efectos aleatorios es como sigue:

$$
\begin{gathered}
\text { status }_{(\mathrm{i}, \mathrm{t})}=\beta_{1} \operatorname{Diftari}_{(\mathrm{i}, \mathrm{t})}+\beta_{2} \\
\text { Emisiones }_{(\mathrm{i}, \mathrm{t})}+\beta_{3} \operatorname{Carbon}_{(\mathrm{i}, \mathrm{t})}+\beta_{4} \\
\text { Fuel }_{(\mathrm{i}, \mathrm{t})}+\beta_{4} \operatorname{Glp}_{(\mathrm{i}, \mathrm{t})}+\beta_{4} \operatorname{Acpm}_{(\mathrm{i}, \mathrm{t})}+\mathrm{v}_{(\mathrm{i}, \mathrm{t})} \\
\operatorname{Con~}_{(\mathrm{i}, \mathrm{t})}=\left(\mathrm{c}_{\mathrm{i}}+\mathrm{u}_{(\mathrm{i}, \mathrm{t})}\right)
\end{gathered}
$$

Vale anotar que la variable EE (energía eléctrica) no se incluye en el modelo que se va a estimar, dado que en pruebas preliminares esta es excluida por problemas de multicolinealidad perfecta.

Respecto a los signos esperados, se anticipa un signo positivo para el estimador de $B_{1}$ toda vez que al aumentar la diferencia de tarifas de los combustibles alternativos frente al gas natural, la probabilidad de realizar la conversión aumenta. Esto refleja explícitamente el comportamiento racional del empresario al minimizar costos de producción. Dado el mayor costo de los combustibles alternativos fuel oil, GLP y ACPM. Frente a la tarifa del gas natural, se esperan signos positivos en los estimadores asociados a estas variables dicotómicas. Respecto al signo de la variable dicotómica carbón, si bien se anticipa podría tomar signo negativo, dado que este combustible es el único de los disponibles con menor precio respecto al gas natural. Por último, se espera un signo positivo del estimador asociado a la variable emisiones, puesto que la normativa ambiental castiga los procesos altamente contaminantes. En este caso, entre mayor sea la carga de contaminación del tipo de combustible utilizado, mayor el incentivo para realizar la conversión industrial a gas natural.

Los resultados de los efectos marginales del modelo de Probit con efectos aleatorios en datos de panel se resumen a continuación:

Tabla 6. Efectos marginales del modelo Probit con efectos aleatorios

\begin{tabular}{cccc}
\hline Variable & Estimador & Desviación Est ${ }^{\star}$ & Significancia \\
\hline Diftari & 0.04348 & 0.0046 & 0.000 \\
\hline Emisiones & 0.00009 & $7.47 \mathrm{e}^{-6}$ & 0.000 \\
\hline Carbón & -1.23169 & 0.083 & 0.013 \\
Fuel & 0.97337 & 0.2605 & 0.000 \\
\hline GLP & 1.25571 & 0.2506 & 0.000 \\
ACPM & 0.59290 & 0.2444 & 0.015
\end{tabular}

* Calculados por el método delta.

Fuente: cálculos del autor con base en estimaciones de STATA12.0 
La opción para calcular los efectos marginales en STATA en un panel es un tanto diferente que para un modelo Probit con datos de sección cruzada o transversal. La opción pu0 calcula la probabilidad asumiendo que el efecto aleatorio para una observación en el panel es igual a cero.

Se observa que todas las variables son significativas, tanto individualmente como en su conjunto (véase la salida del modelo Probit del nivel de significancia del estadístico de Wald). Los resultados son los esperados en cuanto a los signos de los estimadores. Comenzando con la variable diferencia en las tarifas del combustible fósil respecto al gas natural (Diftari), su estimador indica que por un incremento de 1000 pesos en la tarifa, la probabilidad de realizar la transición a gas natural se incrementa en $4.3 \%$. Si bien este estimador probablemente esté sesgado por la existencia de un término de heterogeneidad no observable, el modelo se presenta con el objetivo de estimar los diferenciales en las probabilidades de transición hacia el gas natural por tipo de combustible. Asimismo, se encuentra que entre mayor volumen de emisiones, más proclive está la empresa a realizar la transición a gas natural. Una tendencia que se nota en los últimos años es que los consumidores han incorporado el concepto de consumo responsable, esto es, adquirir productos que minimizan el impacto en el medio ambiente.
Analizando la magnitud de los estimadores que acompañan las variables dicotómicas, sobresale por el tamaño relativo efecto marginal (1.25571) de una empresa que utiliza GLP, siendo este tipo de empresa más proclive a cambiarse a gas natural. Le sigue en probabilidad de sustituir una empresa que utiliza en el momento fuel T-1 como combustible principal con un coeficiente de 0.97 .

Lo anterior contrasta con las empresas que utilizan antes carbón como fuente principal de energía, que tienen la menor probabilidad de conversión. En efecto, si la empresa utiliza carbón antes de la conversión, su probabilidad de realizar la transición es incluso negativa. La explicación reside en el bajo costo del carbón (incluso siendo más barato que el gas) entre todas las alternativas energéticas; siendo el factor costos de producción el más relevante al momento de tomar la decisión realizar la transición a gas natural.

\section{SUSTITUIR O NO SUSTITUIR, HE AHÍ EL DILEMA}

Las industrias que utilicen dentro de sus procesos calderas que quemen petróleos residuales y que tengan acceso al gas natural, pueden optar por cambiar de caldera a otra nueva que lleve incorporado un quemador para gas o, en su defecto, cambiar o adaptar el quemador existente de manera que pueda quemar gas natural. Si bien inicialmente la elección dependerá del 
estado de la caldera o el límite de la capacidad, entre otros, también el ahorro en costos es uno de los determinantes para tal conversión (Dahl \& Matson, 1998).

La mayor participación de combustibles fósiles con altas cargas de emisiones tiene su respuesta en dos factores: la primera son los bajos costos, teniendo en cuenta la tecnología que utiliza el sector industrial del país, la cual no está modernizada; en segundo lugar, lo permisivo de los entes de control del Estado. Aunque un porcentaje del 23 $\%$ puede considerarse bajo, debe anotarse que se puede acercar más al "techo", en la medida en que la industria se percate de la pérdida de competitividad de los combustibles alternativos al gas natural

¿Qué impide el uso intensivo del gas en la industria? Una primera restricción es el desconocimiento de la aplicabilidad del gas natural a diversas actividades productivas en las industrias que utilizan calderas, hornos, marmitas y otros equipos industriales. Adicionalmente se presenta el mito de que se deben hacer grandes inversiones para realizar la sustitución sin un previo análisis del combustible actual versus el gas natural, que permita comparar la calidad calorífica de las distintas alternativas (Axelsson, Harvey $\&$ Berntsson, 2009).

En este orden de ideas, la decisión de las empresas de trasladarse de los demás combustibles a gas natural, dependerá de la competitividad de este combustible respecto a las variables de precio y poder energético. Si el gas no es competitivo en estas variables, las empresas que utilizan carbón y GLP muy seguramente no harán la transición hacia el gas natural. Es poco probable que los empresarios pongan en riesgo la competitividad de la industria haciendo la conversion a gas si este no es competitito en las variables mencionadas. Otras variables para tener en cuenta por el empresario, además del costo del combustible, son el costo inicial de la inversión y el costo de mantenimiento y la evaluacion de la exposicion personal (Rodríguez, 2006).

Antes de revisar a grandes rasgos el proceso necesario para la conversión de las calderas de vapor a gas natural, se requiere adquirir e instalar un sistema de suministro de gas natural que consiste en tuberías (un empalme del distribuidor con los usuarios), trenes de válvulas, medidores, filtros, valvular de alivio, soportes y reductores de presión; todo lo cual debe realizarse bajo estrictas reglas de seguridad. Debe anotarse que los antiguos sistemas de suministro de combustible no necesariamente deben ser anulados, pues pueden ser conservados como respaldo ante un inesperado desabastecimiento de gas natural.

Siguiendo a Chalco (2005), la conversión efectiva a gas natural requiere del desarrollo de la siguiente secuencia: 
1.Revisión de transferencia de calor.

2.Evaluación de la habilidad de los materiales.

3.Estudio de las dilataciones estructurales frente a las nuevas exigencias de temperatura.

4.Revisión y adecuación de los equipos de caldera, tales como el quemador, el ventilador, economizador y controles.

5.Análisis adicionales: revisión de emisiones de contaminantes, capacidad de producción de vapor de la caldera, eficiencia técnica y combustibles de respaldo.

6. Costos de mantenimiento.

\section{Análisis de sustitución}

El costo de conversión a gas natural depende de una serie de variables como el tipo y la capacidad de la caldera; marca y tipo de quemador, trenes de válvula, y otros tipos de accesorios que varían dependiendo de la capacidad de la caldera. Adicionalmente es necesario instalar una estación de reducción de presión y medición de consumo de gas a la entrada de la planta. Finalmente debe tenerse en cuenta la longitud de la tubería, que permita el empalme del distribuidor con el usuario final, así como las tuberías de distribución de gas dentro de la planta.

Dado que cada inversión depende del tipo de caldera y toda vez que esta información no fue suministrada por la fuente de datos primaria (Gases de Occidente), no es posible conocer el costo de inversión del gas natural de cada una de las empresas que realizaron la transición.

A pesar de esto, se cuenta con información sobre el total de la inversión de algunas empresas que utilizan distintos combustibles utilizados, que sin ser representativas del sector que utiliza ya sea GLP, fuel oil u otro tipo de fuente, sí permiten tener una aproximación al tiempo requerido para recuperar la inversión inicial, basados solo en la diferencia del precio de combustibles frente al gas natural.

Hay que anotar que el analisis de sustitucion debe tener en cuenta los costos de mantenimiento relacionados con los equipos que en el momento T-1 utilizaban ACPM, carbón, GLP, o fuel oil. Dichos costos pueden desglosarse en mano de obra interna y externa, repuestos y costos de mantenimientos específicos a cada equipo. Si bien los ahorros en el uso del gas natural en parte se deben a su menor tarifa, tambien debe tomarse en cuenta que los costos de mantenimiento se reducen, pues el mantenimiento por limpieza de los componentes mecanicos es menor en términos absolutos.

Desde el punto de vista de la produccion, el utilizar gas natural tambien muestra varias ventajas operativas, como lo son la disminucion de 
los tiempos improductivos en la planta, debido a fallas en los equipos de ignición en los equipos de combustion, lo cual genera la suspensión de ciertos procesos productivos. En segundo lugar se pueden disminuir los tiempos de paradas de la planta por motivos de limpieza y mantenimiento de los equipos de combustion. Es así como con el uso del gas como combustible principal, la frecuencia de paradas disminuye, no obstante esta fuente tambien requiere de un mantenimiento preventivo, como la revision del sistema de quemadores.

Escenarios del mercado de la energía han sido propuestos por un gran número de autores en la literatura, a menudo vinculados a los estudios de futuros sistemas de energía utilizando modelos energéticos. Sin embargo, a menudo es muy difícil obtener un número de conjuntos coherentes de los datos del mercado de la energía que se pueden utilizar para evaluar nuevas inversiones en sistemas de energía industrial (Axelsson, Harvey \& Berntsson, 2009). Se postula que la mayor parte de los beneficios está basado en la diferencia de los precios corrientes de los combustibles alternativos respecto al gas natural. Claro está que esta diferencia puede cambiar en un contexto dinámico, como lo es el mercado del petróleo, que podría cambiar las demandas de gas en el sector industrial.
El siguiente ejercicio no pretende ser una herramienta adecuada para la generación de tales escenarios, no obstante, ante el vacío que existe sobre el tema, se aborda como una primera aproximación.

A continuación se analizan tres ejemplos de sustitución para los tres combustibles fósiles con alta carga de emisiones ${ }^{8}$. Se toman tres empresas de la muestra, las cuales tienen diferentes requerimientos de energía para su funcionamiento, se utilizan los factores de conversión expuestos en una sección anterior para calcular la cantidad de galones de gas natural para realizar la sustitución. Los precios y costos corresponden al año 2013, y el analisis se realiza con el ACPM, GLP y carbón.

El primer ejercicio de sustitución se realiza con una empresa de la muestra que utiliza ACPM para generar vapor con una caldera. El análisis de conversión permite calcular los requerimientos o demanda de gas natural para obtener la misma energía para cada uno de los combustibles (Painuly \& Parikh,1993).

El ejercicio demuestra que al sustituir el ACPM por el gas natural, el ahorro mensual es del $43 \%$ del costo del equivalente a $\$ 55.364 .151$, mientras que las inversiones que se deben realizar

\footnotetext{
8 Los ejemplos mostrados en este apartado se realizan con empresas de la muestra suministrada por la empresa Gases de Occidente. Por políticas de privacidad se omiten los nombres de las distintas empresas.
} 
El proceso de sustitución de combustibles pesados por gas natural en el sector industrial

Andrés Eduardo Rangel Jiménez • Carlos Johnny Portilla Salazar

Tabla 7. Comparativo de costos combustibles alternativos versus gas natural ${ }^{9}$

\begin{tabular}{|c|c|c|c|c|c|}
\hline Combustible & $\begin{array}{c}\text { Unidad de } \\
\text { Medida }\end{array}$ & \multicolumn{2}{c|}{ Col \$ /Unid. de Medida } & Unidad/Mes & $\begin{array}{c}\text { Valor } \\
\text { costo/mes }\end{array}$ \\
\hline ACPM & Galón & $\$ 8,115.00$ & & 12000 & 97.380 .000 \\
\hline Gas Natural & $\mathrm{m}^{3}$ & & 896 & 46893 & 42.015 .848 \\
\hline Factor de conversión: 3.907724 & \multicolumn{5}{l|}{} \\
\hline GLP & Galón & $\$ 4,200.00$ & & 15000 & 63.000 .000 \\
\hline Gas Natural & $\mathrm{m}^{3}$ & & 896 & 39077 & 35.013 .211 \\
\hline Factor de conversión: 2.605150 \\
\hline Carbón & Kilo & $\$ 210$ & & 300 & 87.000 .000 \\
\hline Gas Natural & $\mathrm{m}^{3}$ & & 510 & 186891 & 95.314 .497 \\
\hline Factor de conversión: 0.622971 & & \\
\hline
\end{tabular}

Fuente: Gases de Occidente S.A. E.S.P.

para este tipo de industria son aproximadamente de $\$ 130.0000 .000$, los cuales se pueden recuperar en aproximadamente 2.4 meses.

Siguiendo con una empresa que utiliza gas licuado de petróleo (GLP) para generar vapor con una caldera, el ejercicio demuestra que al sustituir el GLP por gas natural, el ahorro es del $44 \%$, equivalente a $\$ 27.986 .788$. Las inversiones que se deben realizar para este tipo de industria son aproximadamente de $\$ 95.000 .000$, cuyo tiempo de recuperación se estima en 3.4 meses.

Por último, se analiza la fuente energética que de las tres mencionadas es la que mayor porcentaje de emisiones de, produce. El ejemplo se realizará con una empresa que utiliza carbón para generar vapor con una caldera.

Teniendo en cuenta que para este tipo de empresas, por el alto volumen de gas natural demandado, la Comisión de Regulación de Gas y Energía (CREG) clasifica al usuario como cliente industrial no regulado ${ }^{10}$. El resultado del ejercicio demuestra que al sustituir el carbón por gas natural no hay ahorro, pues el sobrecosto es del orden del $10 \%$, equivalente a una partida adicional de $\$ 8.314 .496$, haciendo aún más inviable esta sustitución.

En este escenario surge el siguiente interrogante: ¿Cómo lograr que este tipo de empresas estén dispuestas a realizar la conversión a gas natural cuando la

9 La unidad de medida corresponde al precio promedio año 2013, a costo asociado de: $\$ 80$

10 CREG: Comisión de Regulación de Energía y Gas: entidad adscrita al Ministerio de Minas y Energía, encargada de la promulgación de la normatividad necesaria para regular los sectores de energía y gas en Colombia. 
principal variable, como son los costos, no favorecen la sustitución? La respuesta está en las alianzas que realizan los comercializadores o distribuidores de gas natural con Ecopetrol (productor) y el TGI (transportador) para garantizar una tarifa atractiva para el cliente, y de este modo permitir la sustitución. Llegar a acuerdos entre los agentes de la cadena del gas natural con el fin de reducir las tarifas finales, es clave en la masificacion del gas como alternativa para las industrias.

Debe anotarse que en la valoración de los distintos combustibles se omiten los costos asociados a la utilización que elevan el costo del energético relacionado con las siguientes actividades:

- Consumo eléctrico por recirculación.

- Costo paradas de mantenimiento.

- Desperdicio y disposición final de residuos.

- Ineficiencia por inquemados.

- Permisos o licencias ambientales.

En el caso del ACPM el costo energético se eleva en un mínimo de $\$ 90$ por galón, mientras que costos asociados a la utilización del GLP se elevan en un mínimo de $\$ 40$ por galón. Un costo adicional relacionado con el ítem de consumo por evaporadores (excluyendo permisos o licencias ambientales y consumo eléctrico por recirculación) debe ser añadido en el caso del GLP.
En la valoración del carbón se deben incluir los costos asociados a su utilización, que elevan el costo del energético en un mínimo de $\$ 80$ por kilogramo relacionado con las actividades anteriormente listadas. Finalmente, la conversion de los equipos a gas natural produce un beneficio ambiental cuantificado aproximadamente de $\mathrm{CO}_{2}$, $\mathrm{CO}$, NOx y material particulado, lo cual permitirá el cumplimiento de regulaciones ambientales por parte del Estado colombiano.

\section{CONCLUSIONES}

Para el sector industrial del Valle del Cauca y norte del Cauca existen ventajas potenciales para los usuarios de calderas de vapor al acceder a un combustible como el gas natural, no solo por su eficiencia y por su relativo bajo precio, sino también por la baja contaminación ambiental que este produce. Cada empresa, al momento de tomar su decisión, deberá sopesar varios factores como el tipo y nivel de consumo actual del combustible, antigüedad de la caldera y capacidad de la misma, y posibles riesgos térmicos al realizar la conversión a gas natural.

La decisión de las empresas de trasladarse de los demás combustibles a gas natural dependerá de la competitividad de este combustible respecto a las variables de precio y poder energético. Seguramente si el gas no es competitivo en estas variables, las empresas que utilizan carbón y GLP no harán 
la transición hacia el gas natural, pues es poco probable que los empresarios pongan en riesgo la competitividad de la industria.

Si bien la diferencia de costos entre el combustible utilizado y el gas, constituye la principal variable al momento de tomar en cuenta seriamente la transición, también lo son el costo inicial de la inversión, el costo de mantenimiento y la evaluación del riesgo de la exposición del personal.

El modelo de Probit con efectos aleatorios para datos de panel permite identificar que la ahorro en costos es una variable significativa al momento de viabilizar un proyecto de sustitucion de combustible. Se confirma que los combustibles menos probables de sustituir son el carbón, el GLP y las biomasas, por el bajo costo de utilización que tienen. En estos casos específicos se deben realizar esfuerzos entre los agentes de la cadena, para buscar un precio más competitivo y a largo plazo, que permita hacer las inversiones necesarias, pues con estas sustituciones se podrían tener unas cifras altas en la reducción de toneladas de $\mathrm{CO}_{2}$.

También, se confirma que la variable emisiones resulta significativa, lo cual es un resultado que se esperaba. En efecto, se presume que la imagen corporativa con una producción limpia y responsable con el medio ambiente se traduzca en gananacias a medio y largo plazo para la empresa, toda vez que los consumidores prefieren consumir productos que sean amigables con el medio ambiente.

Por último, si bien el factor de costos es fundamental al momento de tomar la decisión de reconversión, igualmente es cierto que existen riesgos respecto a la subida del precio del gas natural por efectos de incrementos en el precio del petróleo. Adicionalmente debe tenerse en cuenta que los actuales cuellos de botella respecto a infraestructura están pendientes de solucionar con el fin de abaratar el transporte del gas a sus usuarios finales.

\section{REFERENCIAS}

Axelsson, E., Harvey, S. \& Berntsson, T. (2009). A tool for creating energy market scenarios for evaluation of investments in energy intensive industry.Energy, 34(12), 2069-2074.

Cameron, C. \& Trivedi, P. (2010). Microeconometrics using Stata. USA: The Stata Press. 
Chalco, V. (2005). Beneficios de la conversión a gas natural en calderas de vapor. Recuperado de http://www.unmsm.edu.pe/cedit/linked/analisis $\% 20 \mathrm{de} \% 20$ cambio $\% 20$ de\%20combustible.pdf

Dahl, C. A. \& Matson, T. K. (1998).Evolution of the US natural gas industry in response to changes in transaction costs. Land Economics, 390-408.

Gases de Occidente S.A. ESP (2013). Extraído de la base de datos de Coordinación grandes consumidores, CD-ROM, Febrero 2013.

Harris, M. \& Rogers, M. (2003). Modelling firm innovations using panel probit estimators.Melbourne Institute Working Paper, 20(1).

Hsiao, C. (1986). Analysis of panel data. Cambridge: Cambridge University Press.

IPCC (1996). Revised 1996 IPCC Guidelines for National Greenhouse Gas Inventories: Reference Manual IPCC. U.K.: Bracknell.

Kozulk, R. (2004). La industria del gas natural en América del Sur: situación y posibilidades de integración de los mercados. Chile: Cepal.

Lee, W. (2004). US lessons for energy industry restructuring: based on natural gas and California electricity incidences. Energy Policy, 32(2), 237-259.

Longwell, H. J. (2002). The future of the oil and gas industry: past approaches, new challenges. World Energy, 5(3), 100-104.

Otero, A. (2012). Cali a comienzos del siglo XXI. ¿Crisis o recuperación? Documentos de trabajo sobre economía regional Banco de la Republica, (172). Recuperado de http://mba.americaeconomia.com/sites/mba.americaeconomia.com/files/dtser_172.pdf

Porter, M. (2010). Ventaja competitiva. Creación y sostenibilidad de un rendimiento superior. Madrid: Pirámide.

Painuly, J. P. \& Parikh, J. (1993). Policy analysis of oil substitution by natural gas in India: Transport and industry sectors. Energy policy, 21(1), 43-52.

Rodríguez, M. (2006). Estimación de la demanda de combustibles en República Dominicana. Texto 6. República Dominicana: Unidad de Análisis Económico Secretariado Técnico de la Presidencia.

Wooldridge, J. (2005). Econometric analysis of cross section and panel data. Cambridge, MA: The MIT Press. 
El proceso de sustitución de combustibles pesados por gas natural en el sector industrial Andrés Eduardo Rangel Jiménez • Carlos Johnny Portilla Salazar

\section{ANEXOS}

Anexo 1. Test de heterocedasticidad

\begin{tabular}{|c|c|c|c|c|c|c|}
\hline Source & ss & df & MS & & \multirow{2}{*}{$\begin{array}{l}\text { Number of obs } \\
F(7,3023) \\
\text { Prob }>E\end{array}$} & $\begin{array}{r}=3030 \\
=\quad 550.36\end{array}$ \\
\hline Model & 927.337686 & $7 \quad 132$ & 132.476812 & & & $=0.0000$ \\
\hline Residual & 727.662314 & 3023 & .240708672 & & R-squared & $=0.5603$ \\
\hline Total & 1655 & 3030 & 620462 & & $\begin{array}{l}\text { Adj R-squared } \\
\text { Root MSE }\end{array}$ & $=.49062$ \\
\hline status & Coef. & Std. Err. & $t$ & $p>|t|$ & [958 Conf. & Interval] \\
\hline diftari & .0155066 & .0060782 & 2.55 & 0.011 & .0035887 & .0274244 \\
\hline v18 & $2.89 e-07$ & $3.84 e-08$ & 7.53 & 0.000 & $2.14 e-07$ & $3.64 \mathrm{e}-07$ \\
\hline acpm & .4244574 & .0379694 & 11.18 & 0.000 & .3500089 & .4989059 \\
\hline fuel & .4452339 & .0247154 & 18.01 & 0.000 & .3967731 & .4936947 \\
\hline glp & .5284457 & .0235171 & 22.47 & 0.000 & .4823346 & .5745568 \\
\hline carbon & .3377584 & .0830746 & 4.07 & 0.000 & .17487 & .5006468 \\
\hline ee & .4109048 & .0822044 & 5.00 & 0.000 & .2497226 & .572087 \\
\hline
\end{tabular}

Anexo 2. Probabilidades acotadas entre 0 y 1.

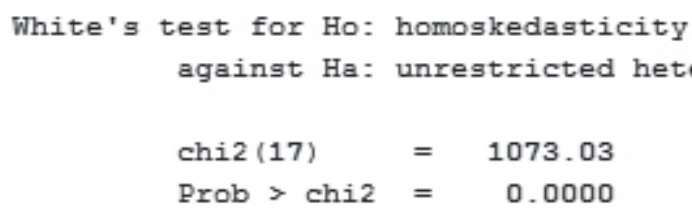

Anexo 3.

sum yhat

\begin{tabular}{r|rrrrr} 
Variable & Obs & Mean & Std. Dev . & Min & Max \\
\hline yhat & 3030 & .5462046 & .0878356 & .3328777 & 2.336972
\end{tabular}


Anexo 4.

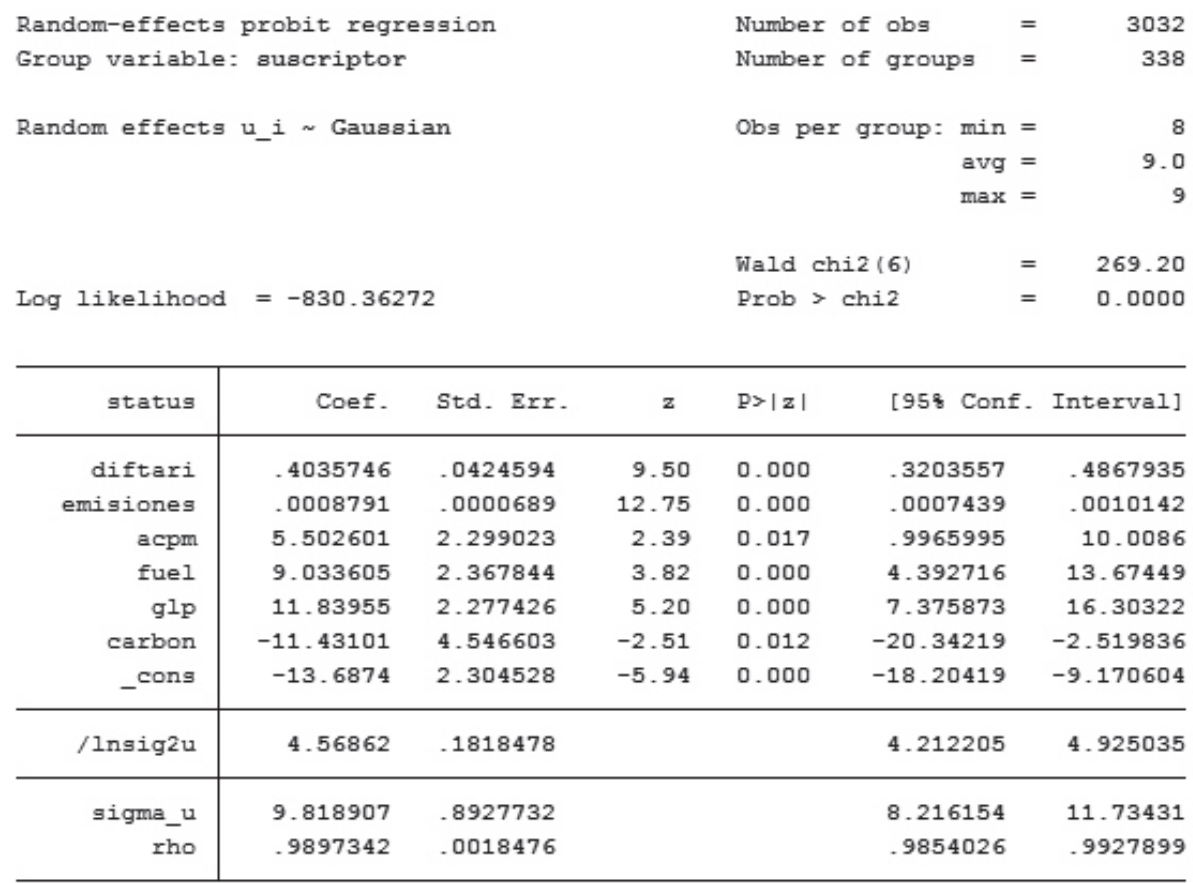

Likelihood-ratio test of rho=0: chibar2 $(01)=1177.02$ Prob $>=\operatorname{chibar2}=0.000$

Anexo 5.

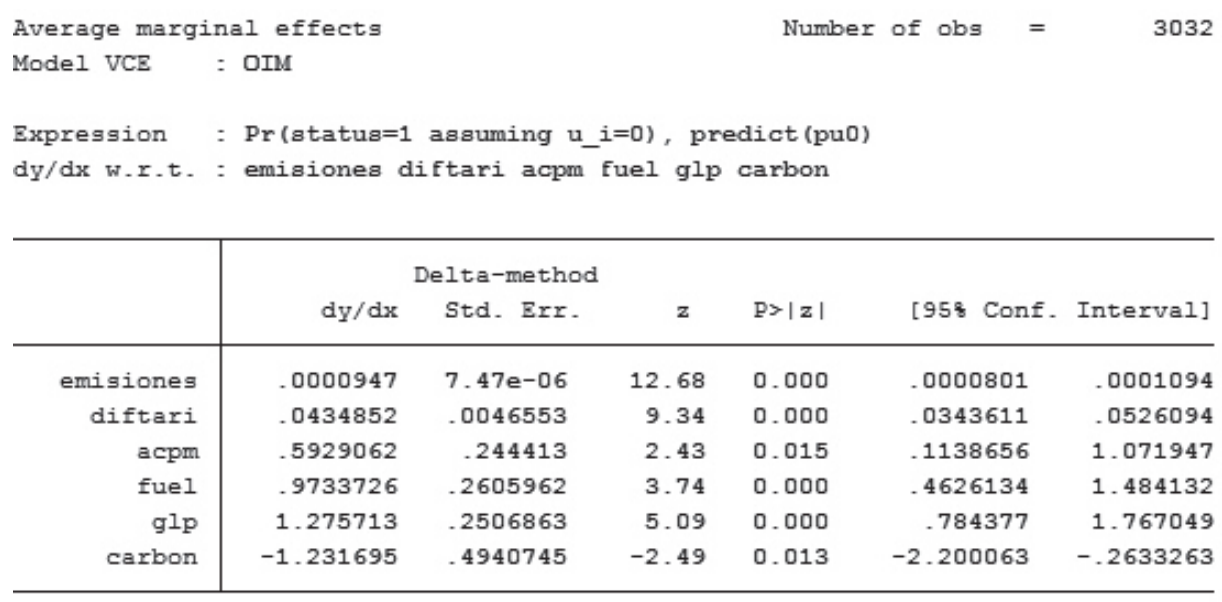


